\title{
Esterilização feminina, AIDS e cultura médica: os casos de São Paulo e Porto Alegre, Brasil
}

\author{
Female sterilization, AIDS, and medical culture \\ in São Paulo and Porto Alegre, Brazil
}

Regina Maria Barbosa 1,2

Daniela Riva Knauth 3,4

\footnotetext{
${ }^{1}$ Núcleo de Estudos Populacionais, Universidade Estadual de Campinas. Av. Albert Einstein 1300, Cidade Universitária, Campinas, $S P$ 13081-970, Brasil. rbarbosa@nepo.unicamp.br 2 Centro de Referência e Treinamento DST/AIDS de São Paulo. Rua Santa Cruz 81, São Paulo, SP 04121-000, Brasil.

3 Núcleo de Pesquisas em Antropologia do Corpo e da Saúde, Programa de Pós-graduação em Antropologia Social, Universidade Federal do Rio Grande do Sul. Av. Bento Gonçalves 9500 , Bloco A2, Porto Alegre, RS 91509-900, Brasil. 4 Departamento de Medicina Social, Faculdade de Medicina, Universidade Federal do Rio Grande do Sul. Rua Ramiro Barcelos 2400,

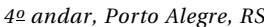
90035-003, Brasil.
}

\begin{abstract}
This paper explores the factors associated with reproductive choices by HIV-positive pregnant women in two settings in Brazil, and particularly how health care providers and the local medical culture can affect these choices. Requesting and submitting to postpartum sterilization provide the focus of the analysis. The data come from a study conducted in prenatal care services in two cities, São Paulo and Porto Alegre. We employed two data collection strategies: collection of information from 427 patient records for HIV-positive women who had received prenatal care, and in-depth interviews with 60 women. While most women in both cities requested tubal ligation after delivery, a much higher proportion of women in Sao Paulo were actually sterilized, as compared to a tiny proportion of women in Porto Alegre. The local medical culture regarding sterilization and organization of prenatal care emerged as important factors for understanding the differences between the two cities.
\end{abstract}

Key words Sexual Sterilization; Acquired Immunodeficiency Syndrome; Women's Health

Resumo Este artigo busca identificar os fatores que interferem nas escolhas reprodutivas de gestantes HIV positivo focalizando, em particular, o papel desempenhado pelos serviços de saúde e pela cultura médica nessas escolhas. O desejo e a realização de laqueadura tubária pós-parto constituem os eventos reprodutivos tomados como questões de análise. Os dados apresentados resultam de uma pesquisa desenvolvida em serviços de pré-natal nas cidades de São Paulo e Porto Alegre, que utilizou um desenho que combinou duas estratégias metodológicas: quantitativa, a partir de dados coletados em 427 prontuários clínicos, e qualitativa, por meio da observação etnográfica e realização de sessenta entrevistas em profundidade. A grande maioria de mulheres nas duas cidades manifestou o desejo de realizar a esterilização depois do parto. Entretanto, uma proporção muito maior de mulheres em São Paulo atingiu sua meta comparada a uma pequena proporção em Porto Alegre. A cultura médica local com relação à laqueadura, aliada à organização da prática de assistência ao pré-natal e parto, mostrou-se fator fundamental para compreender as diferenças encontrada nas duas cidades.

Palavras-chave Esterilização Sexual; Síndrome de Imunodeficiência Adquirida; Saúde da Mulher 


\section{Introdução}

O aumento progressivo da infecção pelo HIV, observado no Brasil entre as mulheres em idade reprodutiva na última década, vem trazendo para a discussão duas questões articuladas. Por um lado, os direitos e as escolhas reprodutivas das mulheres HIV positivo e, por outro, a redução da transmissão vertical do HIV como uma preocupação de saúde pública. Este último aspecto faz com que as mulheres grávidas HIV positivo convivam com um ambiente médico que muda continuamente à medida que novas terapias para tratar o HIV e prevenir a transmissão vertical surgem.

Medidas voltadas à prevenção vertical do HIV vêm sendo progressivamente normatizadas desde o final dos anos 90 no Brasil, entre as quais se destacam: o oferecimento de testagem sorológica para o HIV durante o pré-natal, administração de terapia anti-retroviral à gestante infectada e ao bebê e a substituição do aleitamento natural pelo artificial. Até 2001, o tipo de parto era uma decisão tomada com base em diferentes critérios que eram definidos pela equipe médica e pelos serviços de saúde que atendiam às gestantes HIV positivo, pois se questionavam diversos aspectos com relação ao custo/benefício e a maior morbidade associada à cesariana. Foi apenas em 2001 que se chegou a um consenso com relação ao tipo de parto, ficando a cesariana indicada em um grande número de casos (MS, 2001).

Assim, as gestantes infectadas pelo vírus da AIDS confrontam-se nos serviços de saúde com um conjunto de informações, prescrições e práticas com as quais necessitam, de alguma forma, relacionar-se. Elas precisam aprender a utilizar adequadamente a medicação (tanto a sua como a do bebê), realizar os exames solicitados, aceitar o tipo de parto indicado, etc. De tal forma que é possível postular que toda mudança na prática médica para reduzir a transmissão vertical do HIV modela em algum grau as decisões reprodutivas das mulheres HIV positivo.

Existe um razoável acúmulo de conhecimento referente aos aspectos clínicos da transmissão vertical do HIV, mas pouco se conhece sobre as experiências contraceptivas de mulheres HIV positivo, embora seja possível assumir que em um contexto de altas taxas de esterilização, como o brasileiro, uma grande proporção de mulheres soropositivas que não desejam mais ter filhos também escolheriam ser esterilizadas. Isso é especialmente verdadeiro em um contexto onde o aborto terapêutico é ilegal, exceto nos casos de estupro ou de risco de vida para a gestante. Neste artigo exploramos as escolhas reprodutivas de gestantes HIV positivo em dois cenários no Brasil, de forma a demonstrar como as diferenças entre os serviços de saúde e a cultura médica local, entendida como um conjunto relacionado de idéias e práticas médicas predominantes em um determinado contexto social, bem como o contexto institucional, influem diretamente sobre a possibilidade das mulheres HIV positivo realizarem ou não a esterilização pós-parto.

\section{A esterilização feminina no Brasil e no contexto da AIDS}

Até recentemente a esterilização cirúrgica no Brasil era ilegal, exceto nos casos em que uma gravidez posterior poderia colocar a vida da mulher em risco. Apesar de sua ilegalidade, a esterilização feminina tornou-se no Brasil o método contraceptivo mais popular, usado por $40 \%$ das mulheres casadas entre 15 e 44 anos de idade, com uma proporção significativa dessas esterilizações sendo realizadas por ocasião de uma cesariana (BEMFAM, 1997). Diferenças nessas taxas podem ser observadas em todo o país, variando de $60 \%$ na Região Centro-Oeste a $29 \%$ na Região Sul (BEMFAM, 1997). A existência de diversos discursos e práticas médicas quanto à esterilização e desigualdades no acesso ao planejamento familiar aliada às pressões sociais e econômicas são os principais fatores associados a essas diferenças (Barbosa, 1997).

Com relação à esterilização pós-parto, um estudo conduzido por Potter et al. (2003), em quatro cidades - Porto Alegre, São Paulo, Natal e Belo Horizonte -, entre 1998 e 1999, demonstrou que essa é uma prática muito mais comum nas maternidades privadas $(69 \%)$ do que naquelas pertencentes ao setor público (33\%).

Em 1997, o Congresso Brasileiro aprovou uma lei de planejamento familiar que, pela primeira vez, legalizou a prática da esterilização feminina (e masculina). Em 1999, um conjunto de normas estabelecidas pelo Ministério da Saúde (MS) especificava a implementação da lei estabelecendo que os homens e as mulheres podiam requisitar voluntariamente esterilização cirúrgica, desde que tivessem pelo menos 25 anos de idade ou dois filhos e, se casados, tivessem o consentimento do cônjuge. Adicionalmente, a lei previa um período mínimo de sessenta dias entre o tempo de requerimento e a cirurgia e proibia que as esterilizações fossem realizadas durante o parto, depois de um aborto ou nos 41 dias seguintes ao parto. A nova legislação também possibilitou, pela 
primeira vez, que as clínicas e hospitais fossem reembolsados pelos procedimentos de esterilização voluntária. O sistema público de saúde reembolsaria os custos da esterilização, desde que as clínicas realizassem aconselhamento sobre métodos alternativos de contracepção às pessoas que requisitavam o procedimento. Também estavam previstas na lei penalidades para aqueles que realizassem esterilizações que não se enquadrassem nos critérios estabelecidos.

A lei também define duas condições de "necessidade comprovada" que permitem à mulher realizar esterilização voluntária durante o parto, depois de um aborto ou imediatamente após o parto: mulheres com história de sucessivas cesarianas e/ou portadoras de doenças prévias para quem uma segunda cirurgia ou exposição adicional à anestesia pode apresentar um risco grande à saúde. Nesses casos, a indicação médica deve ser atestada por meio de um documento escrito e assinado por dois médicos (MS, 1999).

No cotidiano dos serviços de saúde, verifica-se que essas duas exceções estão sujeitas às interpretações por parte dos profissionais de saúde, em particular dos médicos, e as influências do contexto institucional no qual eles se encontram inseridos. Considerando-se especificamente o caso de portadora do HIV, cujo acesso à laqueadura não se encontra normatizado, algumas questões se colocam: ser portadora do HIV é considerado uma exceção? Em que situações? Ou exceção é apenas a AIDS? Se a AIDS é uma exceção aceitável para a realização de esterilização pós-parto, é também para todas as pessoas com AIDS, ou somente para aquelas com o sistema imunológico mais comprometido?

\section{As escolhas reprodutivas de mulheres HIV positivo}

Estudos realizados em todo o mundo têm chamado a atenção para a complexidade das interações existentes entre os fatores institucionais, psicossociais e culturais na relação entre escolhas reprodutivas e a epidemia do HIV/ AIDS. Antes da implementação do Protocolo ACTG 076 - que orienta o uso da zidovudina (AZT) durante a gestação e trabalho de parto e nas primeiras seis semanas para o recém nascido -, a maioria dos estudos americanos não mostravam diferenças estatisticamente significativas nas taxas de gravidez e aborto ou nas escolhas contraceptivas de mulheres HIV positivo, quando comparadas a mulheres HIV ne- gativo (Williams et al., 1996). Apenas Lindsay et al. (1995) encontraram uma probabilidade de ser laqueada três vezes maior entre mulheres HIV positivo. Após a implementação do ACTG 076, os estudos americanos que investigaram o impacto do conhecimento dos benefícios do AZT no pré-natal mostraram que essa informação adicional não tinha efeito nas taxas de fertilidade, no uso de contraceptivos ou nas taxas de aborto entre mulheres HIV positivo (Healton et al., 1999; Smits et al., 1999). Somente Bedimo et al. (1998) encontraram diferenças estatisticamente significativas entre mulheres HIV positivo e HIV negativo que incluíram diminuição nas taxas de fertilidade e aumento nas de esterilização para as primeiras.

Já os estudos europeus e australianos anteriores à implementação do Protocolo ACTG 076, ao contrário dos americanos, mostraram consistentemente que as mulheres HIV positivo apresentavam taxas menores de fertilidade, aumento na taxas de esterilização e aumento na incidência da interrupção voluntária da gravidez (De Vincenzi et al., 1997; Lindgren et al., 1998; Stephenson et al., 1996; Thackway et al., 1997). Depois da introdução do Protocolo ACTG 076, estudos longitudinais canadenses e europeus com mulheres HIV positivo continuaram a apresentar taxas menores de fertilidade, mas as taxas de abortos induzidos diminuíram (Hankins et al., 1998; Lindgren et al., 1998; Van Benthem et al., 2000).

Em outros países do mundo, a condição de ser portadora do HIV tem mostrado diferentes efeitos sobre as escolhas reprodutivas das mulheres. Estudos em países africanos, onde o Protocolo ACTG 076 não é amplamente aplicado, não mostraram diferenças entre mulheres infectadas pelo HIV e as mulheres sem o vírus quanto à incidência de gravidez ou uso de contraceptivos (Allen et al., 1993; Nebie et al., 2001).

Por outro lado, estudos com mulheres HIV positivo demonstram claramente o impacto que uma política explícita em favor da laqueadura pós-parto ou de cenários em que esse procedimento é altamente prevalente podem ter nas taxas de esterilização. Na Tailândia, nos hospitais onde a política institucional impõe às mulheres infectadas pelo HIV a adoção de algum método contraceptivo, altas taxas de ligadura tubária $(44,4 \%)$ foram verificadas (Pinchun, 1994). Já no México, cuja cultura contraceptiva é favorável à laqueadura, $61 \%$ de mulheres HIV positivo optaram pela ligadura tubária após o parto (Figueroa-Damian \& Villagrana-Zesati, 2001). Para esses pesquisadores, as elevadas taxas de aceitação de ligadura tu- 
bária encontradas no México, em comparação às encontradas nos estudos americanos e europeus, refletem o alto custo da terapia com AZT para as mulheres nos países em desenvolvimento.

Embora as escolhas reprodutivas feitas pelas mulheres HIV positivo possam diferir no mundo inteiro, estudos qualitativos sugerem que os contextos sociais e culturais são mais determinantes nessas escolhas que o próprio conhecimento do estado sorológico. Os fatores que afetam as escolhas reprodutivas incluem: expectativas de gênero, raça, crenças religiosas, rede de suporte familiar, valor atribuído à maternidade, desejo de ter filhos, disponibilidade da terapia anti-retroviral para reduzir as taxas de transmissão vertical e o apoio do serviço de saúde (Ingram \& Hutchinson, 2000; Kline et al., 1995; Pivnick, 1994). Dentro de cada contexto cultural existem diferentes fatores que interagem de maneira complexa, afetando diferentemente as escolhas reprodutivas feitas pelas mulheres HIV positivo.

Pouco se conhece ainda sobre as escolhas reprodutivas das mulheres HIV positivo no Brasil e como o estado sorológico afeta suas escolhas. Um estudo recente com $1.068 \mathrm{mu}-$ lheres infectadas em São Paulo mostrou que $13 \%$ queriam ter filhos no futuro. Essa proporção foi ainda maior entre mulheres com menos de 30 anos de idade: $39 \%$ das mulheres entre 18 e 24 anos e $25 \%$ entre 25 e 29 anos. A maioria dessas mulheres relacionou seu desejo de ter um filho a um sentimento de "preenchimento como mulher", ao desejo de constituir uma família ou ainda a não ter o número de filhos desejado (Paiva et al., 2002). Um segundo estudo realizado em 1997 com 148 mulheres em um centro de atendimento para DST/AIDS, referência na cidade de São Paulo, revelou que a infecção pelo HIV não alterava o desejo das mulheres de terem mais filhos. Do total, $21 \%$ pensavam em ter mais filhos no futuro, ao mesmo tempo que ter muitos filhos foi o fator associado a não querer ter mais filhos (Santos et al., 2002). E ainda, em trabalho anterior, demonstramos como o diagnóstico de soropositividade para o HIV, bem como as preocupações de evitar a transmissão do vírus ao bebê, direcionam as escolhas femininas no que diz respeito ao tipo de parto desejado (Knauth et al., 2002).

No Brasil, o desejo de uma mulher HIV positivo de ter filhos pode estar reforçado pela queda significativa no risco de transmissão vertical, proporcionado pelo uso de regimes medicamentosos específicos, que, ao contrário de muitos países desenvolvidos, estão disponíveis gratuitamente a todos os brasileiros portado- res do HIV (WHO, 2000). E ainda ao valor atribuído pela cultura brasileira à maternidade, em especial entre os segmentos de menor poder aquisitivo. A família se constitui num dos principais valores do sistema simbólico desses grupos e, como tal, é um elemento fundamental de determinação da identidade social (Bilac, 1978; Duarte, 1986; Fonseca, 1985; Salem, 1981; Sarti, 1996).

\section{A pesquisa: metodologia e universo de investigação}

A fim de explorar o papel exercido pelo contexto institucional e pela cultura médica local nas decisões reprodutivas das mulheres infectadas pelo vírus da AIDS, centraremos nossa análise nas expectativas das mulheres em relação à ligadura tubária e na sua realização, bem como no processo de negociação com a equipe do serviço para a obtenção desse procedimento. Para tal, tomaremos o contexto de duas cidades que apresentam diferenças na prevalência de esterilização feminina: São Paulo e Porto Alegre. Segundo Potter e colaboradores (dados não publicados), na cidade de São Paulo, entre gestantes que não desejavam mais filhos, $24 \%$ das mulheres que deram à luz no setor privado foram laqueadas no pós-parto em comparação a $6 \%$ no setor público. Em contraste, em Porto Alegre, apenas $16 \%$ foram laqueadas no setor privado e $2 \%$ no setor público (dados não publicados). Essa diferença entre as duas cidades é coerente com os dados encontrados pela pesquisa da Sociedade Civil Bem-Estar Familiar no Brasil (BEMFAM, 1997).

Além disso, essas cidades escolhidas pelo fato de apresentarem alta incidência de AIDS, estão entre as 25 cidades com as maiores taxas de incidência de AIDS - 62,0/100 mil em Porto Alegre e 36,9/100 mil em São Paulo (MS, 2002).

Os dados apresentados resultam de uma pesquisa desenvolvida com gestantes HIV positivo, captadas em cada cidade com base em dois contextos institucionais distintos: (1) serviço de pré-natal funcionando dentro ou afiliado a um hospital que tinha um sistema definido de referência para a realização do parto e (2) serviço de pré-natal sem um sistema de referência para o parto num hospital específico. Ao todo foram selecionados três serviços em cada cidade. As clínicas de pré-natal com hospitais de referência escolhidas foram um hospital universitário ou um hospital público de grande porte, e os serviços sem referência para o parto foram aqueles administrados pela prefeitura. Em Porto Alegre, a cidade menor, o pré-natal 
oferecido pelo município é centralizado em um único serviço específico para gestantes HIV positivo. Em São Paulo, principalmente devido à sua grande população e seu tamanho geográfico, o pré-natal está descentralizado em 15 centros de referência específicos para essas gestantes. Desse modo, em São Paulo, foi selecionado um desses centros na Região Nordeste do município.

A pesquisa buscou combinar duas estratégias metodológicas: uma investigação quantitativa, por intermédio da consulta aos prontuários de pré-natal, e uma investigação qualitativa, na qual foram realizadas observação etnográfica e entrevistas em profundidade com as gestantes. Para analisar a taxa de esterilização nos serviços de saúde selecionados, foram coletados dados dos prontuários clínicos de todas as mulheres HIV positivo que fizeram prénatal nesses serviços de julho de 1999 a junho de 2000. Os dados retirados incluíram informações sociodemográficas (tais como idade, escolaridade, número de filhos), o tipo de parto e se ela era esterilizada. Nas situações em que esse dado não constava do prontuário, foi realizada busca ativa dessa informação por meio de contato com as mulheres. Das 422 mulheres atendidas nos serviços investigados no período de um ano e que chegaram ao final da gestação (169 em São Paulo e 258 em Porto Alegre), foram excluídas 25 (10 em Porto Alegre e 15 em São Paulo), visto que não foi possível recuperar a informação sobre o tipo de parto, sobre a ocorrência de esterilização ou ambas as informações. Os casos excluídos não diferiram daqueles da amostra final em termos de média de idade, nível educacional e número de nascimentos vivos. No entanto, as mulheres excluídas, comparadas com aquelas da amostra final, tiveram maior probabilidade de ter realizado o pré-natal em um serviço sem um hospital de referência ( $77 \% v s .45 \%$, respectivamente), o que reflete a qualidade do dado nesse contexto. A amostra final do componente quantitativo é composta, portanto, de 402 gestantes HIV positivo: 154 em São Paulo e 248 em Porto Alegre.

O componente qualitativo da pesquisa é constituído pelas observações etnográficas realizadas nos serviços nos quais as mulheres foram recrutadas e por um conjunto de entrevistas semi-estruturadas, realizadas com sessenta mulheres incluídas na amostra maior acima descrita, trinta em cada cidade. Foram realizadas duas entrevistas com cada mulher: a primeira durante a gravidez e uma segunda após o parto. Visando garantir a diversidade de participantes, os seguintes critérios foram levados em consideração: número de filhos vivos (ne- nhum, um, dois ou mais), idade (abaixo de 25, acima de 25), se ela tinha uma relação estável, estado sorológico do parceiro e tempo de diagnóstico (durante a gravidez, durante gravidez anterior ou em outro período). A primeira entrevista foi realizada entre o terceiro e o sexto mês de gravidez e as entrevistas pós-parto foram realizadas aproximadamente dois meses depois da data do parto. Todas as entrevistas em Porto Alegre foram realizadas na casa da entrevistada; em São Paulo, em razão do tamanho da cidade, a primeira entrevista foi realizada no serviço de pré-natal e a maioria das entrevistas pós-parto na casa da mulher. As entrevistas buscaram apreender as intenções reprodutivas das mulheres HIV positivo, desejos, frustrações e nível de satisfação com os serviços, bem como informações sociodemográficas gerais e dados relativos ao nascimento.

Buscando assegurar o sigilo sobre o diagnóstico, as potenciais participantes eram convidadas, primeiramente, pelos próprios profissionais do serviço e, caso aceitassem participar da pesquisa, eram encaminhadas aos pesquisadores. Todas as mulheres que concordaram em participar do estudo assinaram o termo de consentimento livre e esclarecido. Foi garantido a todas as mulheres que a recusa em participar da pesquisa não teria implicações sobre o acompanhamento e tratamento no serviço de saúde.

Os dados dos prontuários clínicos foram analisados usando o SPSS (versão 8.0). O teste de qui-quadrado foi usado para analisar as diferenças entre as mulheres que foram e as que não foram esterilizadas. Análise de resíduo ajustado foi usada para explorar as tendências de associação entre esterilização e tipo de serviço de pré-natal observados nas duas cidades. Para a análise qualitativa buscou-se evidenciar não apenas as principais categorias acionadas, mas também os significados implicados nos relatos das informantes. Para a sistematização dos dados qualitativos foi utilizado o programa Sphinx Léxica (versão 4.0).

\section{A esterilização de mulheres HIV positivo em São Paulo e em Porto Alegre}

As diferenças nas características sociodemográficas e reprodutivas entre as mulheres nas duas cidades são marcantes. Em Porto Alegre, a amostra era mais jovem do que a correspondente em São Paulo, apresentava menor nível educacional, mais filhos, taxas muito menores de cesariana e uma proporção maior de prénatal em serviços sem um hospital de referên- 
cia. Este último dado reflete o tipo de atendimento às gestantes HIV positivo existente em Porto Alegre: centralizado em um único serviço municipal. As mulheres em ambas as cidades tinham, na sua maioria, deixado a escola antes de completar o segundo grau e possuíam pelo menos dois filhos vivos. Em geral, o perfil do grupo de mulheres entrevistadas segue a mesma tendência observada no grupo maior de onde foram retiradas. O número de mulheres segundo tipo de serviço de pré-natal foi definido pelo desenho do estudo (Tabela 1).

Do total de 402 mulheres atendidas no prénatal no período estudado, $22 \%$ foram esterilizadas depois do parto, sendo possível observar enormes diferenças entre as duas cidades; apenas $4,4 \%$ das mulheres em Porto Alegre foram esterilizadas, contra 50,6\% em São Paulo (Tabela 2).

Em São Paulo, a proporção de esterilização foi maior entre as mulheres com 25 anos ou mais de idade, com menos escolaridade. O número de filhos vivos segue a tendência esperada, em que mulheres com somente um filho apresentam taxas menores de esterilização que aquelas com dois ou mais filhos. E, enquanto a maioria das mulheres que tiveram cesariana foram esterilizadas, entre as que tiveram parto normal aproximadamente 1 em 5 mulheres também foram esterilizadas. Além disso, a proporção de esterilização foi muito maior entre as mulheres atendidas no pré-natal com hospital de referência para o parto. O resultado da análise multivariada realizada para São Paulo, apresentado em outro artigo (Barbosa et al., submetido), confirmou a associação existente entre esterilização e número de filhos vivos, tipo de parto e tipo de serviço de pré-natal.

Em Porto Alegre, como o número de mulheres esterilizadas foi muito pequeno, é difícil estabelecer comparações, mas ainda assim é interessante notar que nenhuma mulher com idade abaixo de 25 anos ou com um filho ou que teve parto normal foi esterilizada, ao passo que em São Paulo encontramos esterilização em mulheres com essas mesmas características. Cabe também destacar que, comparando as mulheres que fizeram pré-natal em um serviço com um hospital de referência e aquelas que tiveram acompanhamento em um serviço sem hospital de referência, a análise de resíduos ajustados nos permite identificar tendências de associação diferentes entre as duas cidades pesquisadas. Enquanto que em São Paulo há uma associação positiva entre esterilização e o pré-natal em serviço com um hospital

Características das mulheres segundo dados obtidos do prontuário e das entrevistas em profundidade.

\begin{tabular}{|c|c|c|c|c|}
\hline \multirow[t]{2}{*}{ Características } & \multicolumn{2}{|c|}{ Porto Alegre } & \multicolumn{2}{|c|}{ São Paulo } \\
\hline & $\begin{array}{l}\text { Dados do } \\
\text { prontuário } \\
(n=248)\end{array}$ & $\begin{array}{l}\text { Entrevistas em } \\
\text { profundidade } \\
(n=30)\end{array}$ & $\begin{array}{l}\text { Dados do } \\
\text { prontuário } \\
(\mathrm{n}=154)\end{array}$ & $\begin{array}{l}\text { Entrevistas em } \\
\text { profundidade } \\
(n=30)\end{array}$ \\
\hline \multicolumn{5}{|l|}{ Idade } \\
\hline$<25$ & 37,2 & 44,4 & 27,9 & 20,0 \\
\hline $25+$ & 62,8 & 55,6 & 72,1 & 80,0 \\
\hline \multicolumn{5}{|l|}{ Nível educacional } \\
\hline 1o grau completo e menos & 88,4 & 86,2 & 69,7 & 66,7 \\
\hline 2 o grau incompleto e mais & 11,6 & 13,8 & 30,3 & 33,3 \\
\hline \multicolumn{5}{|l|}{$\mathrm{Número}$ de filhos } \\
\hline $0-1$ & 19,8 & 13,3 & 28,6 & 20,0 \\
\hline $2+$ & 81,1 & 84,7 & 71,4 & 80,0 \\
\hline \multicolumn{5}{|l|}{ Tipo de parto } \\
\hline Normal & 57,3 & 46,7 & 20,9 & 30,0 \\
\hline Cesariana & 42,7 & 53,3 & 79,1 & 70,0 \\
\hline \multicolumn{5}{|l|}{ Tipo de serviço de pré-natal } \\
\hline Tipo 1 (com referência de parto) & 45,6 & 66,7 & 71,4 & 66,7 \\
\hline Tipo 2 (sem referência de parto) & 54,4 & 33,3 & 28,6 & 33,3 \\
\hline
\end{tabular}


de referência, em Porto Alegre essa associação é negativa, indicando que nessa cidade a ligadura tubária encontra-se associada aos serviços que não contam com um hospital de referência.

\section{Desejo e negociações para a esterilização}

Uma possível explicação para as grandes diferenças nas proporções de mulheres HIV positivo que foram esterilizadas após o parto na cidade de São Paulo é a existência de diferentes níveis de demanda para os procedimentos. Os resultados das entrevistas em profundidade mostram que este definitivamente não é o caso. A intenção de não ter mais filhos foi quase uma unanimidade entre as sessenta entrevistadas, manifestada por 29 mulheres em Porto Alegre e 28 em São Paulo. Entre as principais razões apresentadas pelas mulheres para encerrar a carreira reprodutiva encontram-se a infecção pelo HIV, dificuldades econômicas e já ter o número desejado de filhos. É interessante notar, no entanto, que a infecção pelo HIV aparece como o principal motivo para a grande maioria das mulheres de Porto Alegre, enquanto essa é uma razão alegada apenas por cerca da metade das entrevistadas de São Paulo.

Para a maioria das mulheres que não queriam mais filhos, o desejo de tornar-se esterilizada depois do nascimento - como elas afirmaram nas entrevistas realizadas durante a gravidez - era extremamente forte, tanto em Porto Alegre (21 entrevistadas sobre 29) quanto em São Paulo (22 sobre 28). Assim, ao contrário do que poderíamos supor em função do alto número de mulheres esterilizadas na nossa amostra em São Paulo, como já evidenciado, o desejo de ser esterilizada após o parto é praticamente idêntico nas duas cidades. Observando os resultados, no entanto, vemos uma cena dramaticamente diferente entre as duas cidades. Contrariamente aos seus desejos, em Porto Alegre somente duas mulheres foram esterilizadas, enquanto em São Paulo mais da metade das que manifestaram a intenção de fazer uma laqueadura tubária conseguiram realizá-la.

Este dado sinaliza a influência que o contexto institucional e a cultura médica local exercem sobre as escolhas reprodutivas das mulheres HIV positivo. Os dados das entrevistas, bem como as observações realizadas nos serviços, revelam que em São Paulo há uma maior aceitabilidade dos serviços e mesmo um estímulo destes - especialmente nos serviços com hospital de referência para o parto - no que se re-
Tabela 2

Proporção de mulheres que realizaram esterilização depois do parto, segundo variáveis selecionadas.

\begin{tabular}{|c|c|c|}
\hline Variáveis & $\begin{array}{l}\text { Porto Alegre } \\
(n=248)\end{array}$ & $\begin{array}{l}\text { São Paulo } \\
(\mathrm{n}=154)\end{array}$ \\
\hline Total & 4,4 & 50,6 \\
\hline \multicolumn{3}{|l|}{ Idade } \\
\hline$<25$ & $0,0 *$ & 46,5 \\
\hline $25+$ & 7,1 & 52,3 \\
\hline \multicolumn{3}{|l|}{ Nível educacional } \\
\hline lo grau completo e menos & 4,2 & $57,6^{*}$ \\
\hline 20 grau incompleto e mais & 7,1 & 37,2 \\
\hline \multicolumn{3}{|l|}{ Número de filhos vivos } \\
\hline $0-1$ & $0,0 *$ & $33,3 *$ \\
\hline $2+$ & 6,3 & 77,0 \\
\hline \multicolumn{3}{|l|}{ Tipo de parto } \\
\hline Normal & $0,0^{*}$ & $18,8^{*}$ \\
\hline Cesariana & 10,4 & 59,5 \\
\hline \multicolumn{3}{|l|}{ Tipo de serviço de pré-natal } \\
\hline Tipo 1 (com referência de parto) & 2,7 & $59,1^{*}$ \\
\hline Tipo 2 (sem referência de parto) & 5,9 & 29,5 \\
\hline
\end{tabular}

$* p<0,05$.

fere à demanda pela ligadura tubária. É assim que em um dos serviços investigados a oferta de esterilização pós-parto ocorria já na primeira visita ao serviço, antes mesmo da consulta médica, e nas visitas subseqüentes ao serviço as mulheres eram relembradas da opção de esterilização. O caso de uma entrevistada que, ao salientar que todo o seu trabalho de parto indicava que teria um parto normal, mas que na última hora se encaminhou para uma cesariana, é exemplar da facilitação do serviço para a realização da laqueadura, tanto no sentido de privilegiar a cesariana, quanto na dispensa de um procedimento regular, que é o consentimento do cônjuge para a realização da laqueadura: " $a$ médica ainda falou: 'ela não pode, não pode nascer normal. Porque eu vou fazer a laqueadura nela e vai ter que ser cesariana'. (...) Aí, depois que deu cinco dedos de dilatação, virou, virou. O nenê tava virado pra baixo, pra nascer normal, virou pra cima. E, graças a Deus, nasceu cesariana. (...) Aí eu peguei e pensei também da laqueadura, pensei: 'vou falar da laqueadura, né? Quem sabe ela possa me ajudar, né?. Aí, da laqueadura, ela falou que o meu marido tinha que assinar. Aí, lá foi mais um causo! (...) Como ele vende rosa em farol, então fica difícil (...) Aí, 
minha mãe se ofereceu pra assinar, família, né? Se ofereceu pra assinar. Aí, ela pegou e falou o seguinte: 'tem que ser o marido dela, né? Pra autorizar' (...) sem a assinatura dele não pode, né? - Tá, bom, tudo bem. Pelo menos eu tentei, né? (...) E ela avisou para os médicos que se acontecesse alguma dor assim, se avançasse, né? Que era pra avisar pra ela, porque ela que ia fazer o meu parto. (...) Ela falou assim: 'não, eu decidi, eu mais dois médicos de lá, que eu não sei o nome deles, nós decidimos que vamos fazer a laqueadura em você'. (...) Esses dois médicos assinaram, ela assinou, né? Responsável e depois, eu assinei, depois que eu ganhei" (Entrevistada de São Paulo, 28 anos, dois filhos).

Ou ainda a história relatada por outra entrevista, na qual fica bastante claro uma certa indução da equipe, mesmo contrariando a vontade da paciente, para a escolha da laqueadura como método contraceptivo: "a doutora falou assim: 'olha, a laqueadura é fácil. Você faz a laqueadura'. (...) Eu ia fazer lá, né? (...) É... mas eu não falei que eu não queria ter mais filhos. Eu não lembro o assunto direito, como que entrou nesse assunto, mas eu lembro que ela falou: 'não, eu faço a sua laqueadura' (...) Ai, eu não sei o que eu vou fazer. Ela perguntou assim, como que eu tinha engravidado. Eu falei: 'foi a camisinha'. Eu conversei com o médico que eu queria... um anticoncepcional e ele não me aconselhou. Aí ela falou: 'eu faço a sua laqueadura'. Que aí eu não iria ter mais problemas. Entende? (...) O que todo mundo fala para mim, tanto é que quando eu me recusei a assinar a laqueadura, nenhum médico fala se eu quero ter mais filhos no futuro: 'você tem duas patologias graves, eu não aconselho você a ter mais filhos'. Aí, eu falei pra doutora: 'mas eu não quero ter mais filhos, eu só não quero operar'” (Entrevistada de São Paulo, 30 anos, primeiro filho).

Já na Cidade de Porto Alegre, os serviços tendem a desestimular a laqueadura, seja não oferecendo explicitamente essa possibilidade, seja colocando uma série de empecilhos às mulheres que solicitavam ou simplesmente perguntavam sobre a laqueadura tubária. No serviço de pré-natal sem um hospital de referência, que atende a maioria das gestantes HIV positivo, as solicitações de esterilização eram discutidas em consulta e era mesmo realizado uma espécie de encaminhamento, no qual o médico atestava a vontade da mulher de ser esterilizada. Entretanto, é necessário salientar que esse atestado não tem nenhum valor formal e servia apenas como uma moeda a mais a ser utilizada pela mulher na negociação com a equipe médica responsável pelo parto, como evidencia o relato de uma entrevistada de Porto Alegre cuja solicitação para a realização da laqueadura não foi atendida: "e antes de abrir [para a cesariana] eu disse assim, que eu quero entregar esse papel aqui pra vocês que o meu médico mandou. Aí eles leram ali aquele papel que o doutor [médico do pré-natal] mandou, né? Leram, aí daqui a pouco mais ligaram lá não sei pra onde, pro médico eu acho, né? E o médico disse que não, que não adiantava aquele papel pra eles" (Entrevistada de Porto Alegre, 26 anos, 4 filhos).

Assim, embora em ambas as cidades o desejo das mulheres pela esterilização seja praticamente o mesmo, fica evidente que em Porto Alegre há uma cultura médica bastante resistente à realização da laqueadura, tanto que desconhece a nova legislação sobre o assunto, como atestam as observações dos serviços. A infecção pelo vírus HIV não age como elemento facilitador dessa prática, como ocorre em São Paulo, onde as mulheres HIV positivo são mesmo incitadas a se esterilizar. Essa cultura médica encontra-se também disseminada entre as próprias mulheres, visto que muitas delas nem chegam a explicitar o desejo de laqueadura por perceberem que a equipe médica se posicionará contrariamente. A esperança de obter uma ligadura tubária em Porto Alegre se dá mediante a também esperança de fazer uma cesariana, seja em função da redução do risco de transmissão vertical ou de alguma intercorrência do próprio parto, mesmo que isso implique o medo e a angústia, como expressa uma entrevistada: "Não sei, o meu medo é fazer cesariana, porque, aí não sei como que é, eu nunca fiz (...) Mas eu acho que eu vou ter que pedir [uma cesariana], né? Porque quero fazer o desligamento, (...) fazer cesariana.(...) Mas eu não queria fazer cesariana pra desligar, mas eu vou ser obrigada fazer..." (Entrevistada de Porto Alegre, 30 anos, 1 filho).

Contudo, toda a determinação das mulheres em se submeterem a procedimentos que não gostariam, como uma cirurgia e uma anestesia, a fim de obter uma esterilização não é suficiente para que seus desejos e decisões sejam respeitados. Em Porto Alegre, a cultura médica e o contexto institucional afirmam, por intermédio de suas práticas, que mesmo não desejando mais filhos, o melhor método contraceptivo para as mulheres HIV positivo é o uso do preservativo, combinado, às vezes, a algum anticoncepcional hormonal. Já em São Paulo, na perspectiva dos serviços, a esterilização é a melhor solução para as mulheres infectadas pelo vírus da AIDS que não querem mais engravidar. 


\section{Discussão}

Há relativamente poucos estudos abordando o aspecto da esterilização entre mulheres HIV positivo e nenhum foi realizado no Brasil. Embora os resultados do presente estudo não possam ser generalizados para toda a população de gestantes HIV positivo no Brasil, eles indicam vários aspectos que merecem atenção. As mulheres HIV positivo tendem a ter taxas maiores de esterilização que as não infectadas, pelo menos nessas duas áreas metropolitanas, fato também identificado pela maioria dos estudos internacionais. Um estudo semelhante, realizado em 1998/1999 - com 1.612 gestantes atendidas em pré-natal em serviços disponíveis para a população em geral -, mostrou que, entre as mulheres que não queriam mais filhos e que realizaram o parto na rede pública de saúde, $2 \%$ foram esterilizadas depois do parto em Porto Alegre e 6\% em São Paulo (Potter, dados não publicados). No presente estudo, nós encontramos, no universo total de gestantes atendidas nos serviços selecionados, mais de $4 \%$ das mulheres HIV positivo em Porto Alegre esterilizadas após o parto em comparação a $51 \%$ em São Paulo - dados que sugerem portanto uma associação positiva entre ser infectada com HIV e ser esterilizada.

Mesmo o desejo de tornar-se esterilizada sendo alto entre essas mulheres e semelhante nas duas cidades, uma mulher tem menos probabilidade de ser esterilizada em Porto Alegre que em São Paulo. Além disso, em Porto Alegre, onde as mulheres são bem conscientes da dificuldade de realizar a esterilização pós-parto, elas dão grande ênfase ao seu estado sorológico como motivo para não querer mais filhos e para escolher a esterilização, sugerindo uma esperança de que o seu estado sorológico possa trazer-lhes uma "vantagem" adicional para convencer o médico a fazer a ligação tubária.

Esses resultados revelam que para entender as decisões reprodutivas das mulheres no contexto do HIV/AIDS nós devemos considerar outros aspectos, e não apenas os desejos e decisões individuais. Alguns autores têm apontado nessa direção e proposto esquemas mais complexos para a compreensão das diferentes dimensões que determinam as práticas sociais. Bajos \& Marquet (2000), refletindo sobre os dados dos grandes estudos quantitativos desenvolvidos na Europa nos últimos anos sobre comportamento sexual da população, sugerem contextualizar as decisões e práticas dos indivíduos, para fins de análise (já que na prática todos os níveis encontram-se extremamente interligados), em quatro níveis: (1) o contexto ins- titucional e macrossocial; (2) o contexto mais limitado do relacionamento social; (3) o contexto da própria interação entre os parceiros; e (4) o nível intrapessoal.

O primeiro nível seria o mais estrutural, no qual se enquadrariam os aspectos sócio-econômicos mais amplos, mas também aqueles de ordem valorativa, como por exemplo, o gênero. O segundo seria um nível intermediário, que poderíamos pensar como o contexto sócio-cultural local, que determina um conjunto mais restrito de valores e significados. O terceiro nível seria aquele no qual se situam os relacionamentos interpessoais propriamente ditos, isto é, as relações sociais. Por fim, o último nível seria aquele mais restrito às características propriamente individuais, tais como personalidade, história de vida, experiências.

Desse modo, analisando as decisões reprodutivas no contexto das gestantes HIV positivo, em especial aquelas relacionadas à esterilização, é fundamental pensarmos nesses diferentes níveis na medida em que nesse contexto específico estão em questão aspectos que ultrapassam, em muito, o nível de decisão individual. Assim, se num primeiro momento devemos considerar a importância da cultura na valorização da maternidade, outros níveis parecem agir nas decisões a serem tomadas pelas mulheres durante e após a gestação. No caso das mulheres infectadas pelo HIV, as decisões reprodutivas aparecem claramente influenciadas pela cultura médica local referente à esterilização e pela forma de organização da prática assistencial com relação ao pré-natal e parto a qual essas mulheres estão vinculadas.

Mais especificamente, temos de considerar a relação entre a cultura médica local e a organização da prática do cuidado em saúde. Para explorar esse aspecto, vamos assumir que os serviços de pré-natal com um hospital de referência para o parto são mais organizados que os serviços de pré-natal sem um hospital de referência. Em São Paulo, onde a cultura médica favorece a esterilização feminina, mulheres que receberam cuidado pré-natal em um sistema de saúde mais organizado tiveram maior probabilidade de receber um procedimento de esterilização que aquelas atendidas em um serviço menos organizado. Em Porto Alegre, onde a cultura médica é mais resistente à esterilização feminina, as mulheres que receberam cuidado pré-natal em um sistema similar mais organizado tiveram menor probabilidade de receber um procedimento de esterilização. Essa relação inversa pode ser um reflexo das duas culturas médicas diferentes com relação à esterilização presentes em São Paulo e em Por- 
to Alegre. Resumindo, poderíamos dizer que quanto mais organizada é a prática de cuidado de saúde mais ela reproduz a cultura médica local.

Em São Paulo a cultura médica encoraja as mulheres a realizar a esterilização, enquanto em Porto Alegre essa prática é desestimulada. Isto significa que em Porto Alegre muitas mulheres HIV positivo podem ter frustadas suas tentativas de tornar-se esterilizadas, apesar de seu forte desejo de realizar a esterilização. Em São Paulo, onde as mulheres soropositivas claramente têm mais possibilidade de tornar-se esterilizadas, elas podem enfrentar o outro lado do problema: o corpo clínico pode encorajar as mulheres a realizar a esterilização para excluir a possibilidade de outros métodos.

Em São Paulo, onde a cultura médica é favorável à esterilização feminina, as mulheres HIV positivo são liberadas do cumprimento da lei com relação à proibição de esterilizações pós-parto, com base na interpretação de que um segundo ato cirúrgico é considerado um risco significativo para a mulher. Como não existe consenso ou normas para tal procedimento, cada serviço de saúde acaba desenvolvendo os seus próprios padrões de acordo com o seu entendimento das normas. Além disso, em São Paulo nós constatamos que os critérios para a realização de esterilizações foram aplicados de uma maneira mais permissiva, realizando-se, por exemplo, esterilizações em mulheres com menos de 25 anos de idade depois do nascimento de seu primeiro filho, ou ainda realizando o procedimento sem o consentimento do cônjuge.

Em Porto Alegre, onde a cultura médica desencoraja a esterilização, os desejos das mulheres de se esterilizarem são frustrados. Nos poucos casos em que a ligadura tubária foi realizada, ela foi decidida no momento do parto e, na maioria dos casos, foi realizada nos serviços menos estruturados. Assim, nossos dados indicam que as chances da mulher fazer uma esterilização são quase inteiramente função do serviço de pré-natal que ela freqüenta, e, por sua vez, da cultura médica local relativa à esterilização.

Um comentário final deve ser feito sobre as diferenças observadas entre nossos resultados e aqueles encontrados em outros estudos no Brasil com relação ao desejo das mulheres HIV soropositivas de não quererem mais filhos. Paiva et al. (2002) encontraram que $13 \%$ das mulheres queriam ter mais filhos no futuro, enquanto Santos et al. (2002) verificaram uma proporção maior, $21 \%$. Mesmo considerando que em nossos dados somente 3 de 60 mulhe- res relataram desejo de ter mais filhos e que esses dados são baseados em uma pequena amostra qualitativa, podemos sugerir que essas diferenças apontam para a necessidade de investigar o significado dos desejos das mulheres HIV positivo de serem esterilizadas, no sentido de compreender o impacto que o viver com HIV/AIDS tem em seu desejo de esterilização enquanto estão grávidas. A gravidez em si evoca um certo grau de ansiedade e incerteza que pode ampliar a pressão dessas mulheres na direção da laqueadura.

\section{Conclusão}

As decisões referentes ao âmbito reprodutivo envolvem sem dúvida aspectos altamente sensíveis e complexos. Os fatores que moldam as escolhas das mulheres, quando elas enfrentam a infecção do HIV, no seu dia-a-dia são ainda mais complexos. As mulheres HIV positivo tendem a ter taxas maiores de esterilização que as não infectadas. A cultura médica local e a posição do serviço de saúde com relação à esterilização, que sustenta diferentes interpretações da lei no Brasil, são reforçadas por préconcepções com relação à AIDS. Aconselhamento, apoio para planejamento familiar e uma compreensão ampla da complexidade envolvida nas escolhas reprodutivas por parte dos serviços e profissionais de saúde devem ser aprimorados, para que as mulheres possam tomar sua própria decisão. Para que isso seja possível, não é suficiente simplesmente atender (ou não) os desejos de esterilização das mulheres, mas compreender as circunstâncias complexas nas quais essas decisões são tomadas e apoiadas (ou não) pelos serviços de saúde, o que implica focar não apenas as características e desejos individuais das mulheres HIV positivo, mas também as políticas e as práticas dos profissionais e serviços de saúde. 


\section{Agradecimentos}

Kristine Hopkins e Joseph Potter, pesquisadores que compuseram a equipe de coordenação do estudo, do Population Research Center da University of Texas at Austin; Maia Hightower, bolsista do Rotary Internacional, pela realização da revisão da literatura; Regina Facchini e Marion Pegorario, auxiliares de pesquisa. Este estudo foi financiando pelo US National Institutes of Health (R01 HD 33761-13) e pelo Conselho Nacional de Desenvolvimento Científico e Tecnológico.

\section{Referências}

ALLEN, S.; SERUFILIRA, A.; GRUBER, V.; KEGELES, S.; VAN DE PERRE, P.; CARAEL, M. \& COATES, T. J., 1993. Pregnancy and contraception use among urban Rwandan women after HIV testing and counseling. American Journal of Public Health, 83:705-710.

BAJOS, N. \& MARQUET, J., 2000. Research on HIV sexual risk: Social relations-based approach in a cross-cultural perspective. Social Science and $\mathrm{Me}$ dicine, 50:1533-1546.

BARBOSA, R. M., 1997. Negociação Sexual ou Sexo Negociado: Gênero, Sexualidade e Poder em Tempos de AIDS. Tese de Doutorado, Rio de Janeiro: Instituto de Medicina Social, Universidade do Estado do Rio de Janeiro.

BARBOSA, R. M.; HOPKINS, K.; KNAUTH, D. R. \& POTTER, J. E., submetido. The impact of health care providers on female sterilization among HIV-positive women in Brazil. Social Science and Medicine.

BEDIMO, A. L.; BESSINGER, R. \& KISSINGER, P., 1998. Reproductive choices among HIV-positive women. Social Science and Medicine, 46:171-179.

BEMFAM (Sociedade Civil Bem-estar Familiar no Brasil), 1997. Pesquisa Nacional Sobre Demografia e Saúde 1996. Calverton: Macro International/BEMFAM.

BILAC, E., 1978. Famílias de Trabalhadores: Estratégias de Sobrevivência. São Paulo: Símbolo.

DE VINCENZI, I.; JADAND, C.; COUTURIER, E.; BRUNET, J. B.; GALLAIS, H.; GASTAUT, J. A.; GOUJARD, C.; DEVEAU, C. \& MEYER, L., 1997. Pregnancy and contraception in a French cohort of HIV-infected women. SEROCO Study Group. AIDS, 11:333-338.

DUARTE, L. F. D., 1986. Da Vida Nervosa nas Classes Trabalhadoras. Rio de Janeiro: Zahar.

FIGUEROA-DAMIAN, R. \& VILLAGRANA-ZESATI, R., 2001. Factors associated with acceptance of postpartum tubal ligation among HIV-infected women. Salud Pública de México, 43:97-102.
FONSECA, C., 1985. Valeur marchande, amour maternel et survie: Aspectos de la circulation des enfants dans en bindonville Brésilien. Annales ESC, 5:991-1022.

HANKINS, C.; TRAN, T. \& LAPOINTE, N., 1998. Sexual behavior and pregnancy outcome in HIV-infected women: Canadian women's HIV study group. Journal of Acquired Immune Deficiency Syndrome and Human Retrovirology, 18:479-487.

HEALTON, C.; TAYLOR, S.; MESSERI, P.; WEINBERG, G. \& BAMJI, M., 1999. Effects of ZDV-based patient education on intentions toward ZDV use, HIV testing and reproduction among a US cohort of women. AIDS Care, 11:675-686.

INGRAM, D. \& HUTCHINSON, S. A., 2000. Double binds and the reproductive and mothering experiences of HIV-positive women. Quality Health Research, 10:117.

KLINE, A.; STRICKLER, J. \& KEMPF, J., 1995. Factors associated with pregnancy and pregnancy resolution in HIV seropositive women. Social Science and Medicine, 40:1539-1547.

KNAUTH, D. R.; BARBOSA, R. M.; HOPKINS, K.; PEGORARIO, M. \& FACHINI, R., 2002. Cultura médica e decisões reprodutivas entre mulheres infectadas pelo vírus da AIDS. Interface - Comunicação, Saúde, Educação, 11:39-54.

LINDGREN, S.; OTTENBLAD, C.; BENGTSSON, A. B. \& BOHLIN, A. B., 1998. Pregnancy in HIV-infected women: Counseling and care -12 years' experiences and results. Acta Obstetricia et Gynecologica Scandinavica, 77:532-541.

LINDSAY, M. K.; GRANT, J.; PETERSON, H. B.; WILLIS, S.; NELSON, P. \& KLEIN, L., 1995. The impact of knowledge of human immunodeficiency virus serostatus on contraceptive choice and repeat pregnancy. Obstetrics and Gynecology, 85:675679.

MS (Ministério da Saúde), 1999. Portaria SAS/MS-48, de 11/2/99 que Regulamenta a Lei Federal 9263. Brasília: MS. 
MS (Ministério da Saúde), 2001. Guia de Tratamento; Recomendações para a Profilaxia da Transmissão Vertical do HIV e Terapia Anti-retroviral em Gestantes. Brasília: MS.

MS (Ministério da Saúde), 2002. Boletim Epidemiológico AIDS, 16(1).

NEBIE, Y.; MEDA, N.; LEROY, V.; MANDELBROT, L.; YARO, S.; SOMBIE, I.; CARTOUX, M.; TIENDREBEOGO, S.; DAO, B.; OUANGRE, A.; NACRO, B.; FAO, P.; KY-ZERBO, O.; VAN DE PERRE, P. \& DABIS, F., 2001. Sexual and reproductive life of women informed of their HIV seropositivity: A prospective cohort study in burkina faso. Journal of Acquired Immune Deficiency Syndrome, 28: 367-372.

PAIVA, V.; LATORRE, M. R.; GRAVATO, N.; LACERDA, R. \& ENHANCING CARE INITIATIVE - BRAZIL, 2002. Sexualidade de mulheres vivendo com HIV/ AIDS em São Paulo. Cadernos de Saúde Pública, 18:1609-1620.

PINCHUN, P., 1994. Management of HIV infected pregnant women in Chonburi Hospital. Journal of the Medical Association of Thailand, 77:213.

PIVNICK, A., 1994. Loss and regeneration: Influences on the reproductive decisions of HIV positive, drug-using women. Medical Anthropology, 16:3962.

POTTER, J. E.; PERPÉTUO, I. H.; BERQUÓ, E.; HOPKINS, K.; LEAL, O. F.; CARVALHO-FORMIGA, M. C. \& SOUZA, M. R., 2003. Frustrated demand for postpartum female sterilization in Brazil. Contraception, 67:385-390.

SALEM, T., 1981. Mulheres faveladas: Com vendas nos olhos. Perspectivas Antropológicas da Mulher, $1: 3-34$.
SANTOS, N. J. S.; BUCHALA, C. M.; FILIPE, E. V.; BU GAMELLI, L.; GARCIA, S. \& PAIVA, V., 2002. Mulheres HIV positivas, reprodução e sexualidade. Revista de Saúde Pública, 36(4 Sup.):12-23.

SARTI, C. A., 1996. A Família como Espelho: Um Estudo sobre a Moral dos Pobres. Campinas: Autores Associados.

SMITS, A. K.; GOERGEN, C. A.; DELANEY, J. A.; WILLIAMSON, C.; MUNDY, L. M. \& FRASER, V. J., 1999. Contraceptive use and pregnancy decision making among women with HIV. AIDS Patient Care and STDs, 13:739-746.

STEPHENSON, J. M.; GRIFFIOEN, A. \& THE STUDY GROUP FOR THE MEDICAL RESEARCH COUNCIL COLLABORATIVE STUDY OF WOMEN WITH HIV, 1996. The effect of HIV diagnosis on reproductive experience. AIDS, 10:1683-1687.

THACKWAY, S. V.; FURNER, V.; MIJCH, A.; COOPER, D. A.; HOLLAND, D.; MARTINEZ, P.; SHAW, D.; VAN BEEK, I.; WRIGHT, E.; CLEZY, K. \& CALDOR, J. M., 1997. Fertility and reproductive choice in women with HIV-1 infection. AIDS, 11:663-667.

VAN BENTHEM, B. H.; DE VINCENZI, I.; DELMAS, M. C.; LARSEN, C.; VAN DEN HOEK, A. \& PRINS, M., 2000. Pregnancies before and after HIV diagnosis in a European cohort of HIV-infected women: European study on the natural history of HIV infection in women. AIDS, 14:2171-2178.

WILLIAMS, H. A.; WATKINS, C. E. \& RISBY, J. A., 1996. Reproductive decision-making and determinants of contraceptive use in HIV-infected women. Clinical Obstetrics and Gynecology, 39:333-343.

WHO (World Health Organization), 2000. Report on the Global HIVIAIDS Epidemic. Geneva: WHO.

Recebido em 9 de outubro de 2003

Aprovado em 31 de outubro de 2003 